\title{
ILAE Classification of Seizures Types and Epilepsies (2017) \\ Kyaw Linn
}

Department of Pediatrics, Yangon Children's Hospital, Pyidaungzu Yeiktha Street, Yangon, Myanmar

In 2017, for the first time in almost three decades, the International League Against Epilepsy (ILAE) has approved a new classification system for seizures. New classification of seizure types, largely based upon the existing classification formulated in 1981 and also epilepsy types, epilepsy syndromes were published online March 9 in Epilepsia [1-3]. This publication includes three articles related to the new classification, developed through a process initiated by the International League Against Epilepsy (ILAE) in 2010 (ILAE classification of the epilepsies, Operational classification of seizure types by theInternational League Against Epilepsy, Instruction manual for the ILAE 2017 operational classification of seizure types). The new system formally recognizes some seizure types, provides additional information on causes, comorbid conditions and replaces controversial words and terms with more practical terminologies.

Sameer M. Zuberi, Chair of the ILAE Commission for Classification \& Terminology and Emilio Perucca, President of the International League Against Epilepsy remarked that these articles in this issue should beregarded as representing one body of work that has beenpresented in three papers to allow the depth of detail andexplanation necessary for changes that are so fundamentalto the care of people with epilepsy [4].

Actually, seizure classification can be based on many parameters such as pathophysiology, etiology, semiology, network, drug response, age of onset, etc. However, fundamental knowledge of every parameter is not perfect. In the absence of fundamental knowledge, ILAE chose to extend the existing classification into this new classification. This is an operational (practical) system, not a true scientific classification and this classification is predominantly for clinicians, everywhere, either in resource poor setting or in well developed countries.

Since I was a medical student, all medical doctors were quite familiar with old classification of seizure types - generalized seizures (tonic, clonic, tonic clonic, atonic, myoclonic, absence) and partial seizures (simple and complex partial seizures). However, for each and every patient with seizure, we cannot get an accurate history from the witness as well as from the patients for many reasons. Therefore, many clinical seizures types were not so accurate. Since 1981, there were a number of proposals, axis, and changes in original classification, but this 2017 classification is most practical, systematic and holistic.

In the new classification, seizure types are classified depending on the origin of onset, into focal onset, generalized onset, and unknown onset. The origin of onset might not be seen in each and every case of seizure and allowing unknown onset officially makes medical professionals more comfortable and more legal.

In some settings, classification according to seizure type can be the only classification as there may be no access to EEG, video and imaging studies. This is especially important for resource poor settings. Another major change is that seizures formerly known as simple partial are now called focal aware seizure and complex partial seizures are now focal impaired awareness seizures. That makes seizure types more simple and applicable and all medical professionals can understand more about terminologies.

\section{Publication History:}

Received: June 01, 2017

Accepted: June 28, 2017

Published: June 30, 2017

\section{Keywords:}

Epilepsia, Seizures, Classification, Management

Previously, tonic, clonic, myoclonic seizures were generalized seizures, but we all know that there may be a focal tonic seizure, or a focal clonic seizure with jerking, or focal myoclonic seizure. The 2017 classification recognizes these seizure types can be under focal onset.

Consciousness is a complex phenomenon, with both subjective and objective components. Surrogate markers for consciousness usually comprise measurements of awareness, responsiveness, memory, anda sense of self as distinct from others. Retrospective determination of state of consciousnesscan be difficult. The Task Force adopted state of awareness as a relatively simple surrogate marker for consciousness and employed an operational definition of awareness as knowledge of self and environment. I personally feel that this operational definition of consciousness is still not practical and not applicable in pediatric population and we need to find out more suitable surrogate markers.

In the new classification, framework for classifications of epilepsies also emphasize two most important things, co-morbidities and etiologies for the purpose of holistic approach of management. We, medical doctors, especially in busy clinics, tend to forget about these essential issues and pay attention only to seizures control. The new classification framework highlights that seizure control only is not the key management and treating the whole patient is the most important. According to new classification, an etiological diagnosis should be considered from when the patient first presents and at each step along the diagnostic pathway.

Determining comorbidities is another important step in the classification system. There is increasing awareness that many of the epilepsies are associated with comorbidities such as learning, psychological, and behavioral problems.

Terminologies in previous classifications are a bit confusing to public and even to medical doctors. Another novel element is that public opinion was considered in developing the new classification. During the long process of this new classification, ILAE task force also listened to comments from public and as a consequence of this democratic process; new classification is quite helpful for those involved in managing people with epilepsy, including doctors, researchers and even third party payers [5].

"Corresponding Author: Dr. Kyaw Linn, Department of Pediatrics, Yangon Children's Hospital, Pyidaungzu Yeiktha Street, Yangon, Myanmar; E-mail: linnkyaw.neuro@gmail.com

Citation: Linn K (2017) ILAE Classification of Seizures Types and Epilepsies (2017). Int J Pediatr Neonat Care 2: 126. doi: https://doi.org/10.15344/24552364/2017/126

Copyright: (c) 2017 Linn. This is an open-access article distributed under the terms of the Creative Commons Attribution License, which permits unrestricted use, distribution, and reproduction in any medium, provided the original author and source are credited. 
Citation: Linn K (2017) ILAE Classification of Seizures Types and Epilepsies (2017). Int J Pediatr Neonat Care 2: 126. doi: https://doi.org/10.15344/24552364/2017/126

Page 2 of 2

In summary, we, as pediatric neurologists, especially from resource poor setting, welcome this new classification of seizure types and epilepsy types. However, as knowledge grows and concepts evolve, we have to accept and expect that classifications will change. This must be balanced against the requirement for consistency over time and a new emerging knowledge.

\section{References}

1. Scheffer IE, Berkovic S, Capovilla G, Connolly MB, French J, et al. (2017) ILAE classification of theepilepsies: Position paper of the ILAE Commission for Classification and Terminology. Epilepsia 58: 512-521.

2. Fisher RS, Cross JH, French JA, Higurashi N, Hirsch E, et al. (2017) Operational classification of seizure types by the International League Against Epilepsy: Position Paper of the ILAE Commission for Classification and Terminology. Epilepsia 58: 522-530.

3. Fisher RS, Cross JH, D'Souza C, French JA, Haut SR, et al. Instruction manual for the ILAE 2017 operational classification of seizure types. Epilepsia 58: 531-542.

4. Zuberi SM, Perucca E. (2017) Commentary: a new classification is born. Epilepsia 58: 511.

5. Anderson P. (2016) New ILAE classification system for seizures. Medscape medical news. 